\title{
Envelhecimento e dependência no Brasil: características sociodemográficas e assistenciais de idosos e cuidadores
}

\author{
Aging and dependence in Brazil: sociodemographic \\ and care characteristics of older adults and caregivers
}

Roger Flores Ceccon (https://orcid.org/0000-0002-0846-1376) ${ }^{1}$

Luiza Jane Eyre de Souza Vieira (https://orcid.org/0000-0002-5220-027X) ${ }^{2}$

Christina César Praça Brasil (https://orcid.org/0000-0002-7741-5349) ${ }^{2}$

Konrad Gutterres Soares (https://orcid.org/0000-0002-5361-9419) ${ }^{3}$

Virgínia de Menezes Portes (https://orcid.org/0000-0001-6604-1962) ${ }^{1}$

Carlos Alberto Severo Garcia Júnior (https://orcid.org/0000-0003-3367-4151) ${ }^{1}$

Ione Jayce Ceola Schneider (https://orcid.org/0000-0001-6339-7832) ${ }^{1}$

Antonio Augusto Ferreira Carioca (https://orcid.org/0000-0002-1194-562X) ${ }^{2}$
${ }^{1}$ Escola de Saúde Coletiva, Universidade Federal de Santa Catarina. Rodovia

Governador Jorge Lacerda 3201, Urussanguinha. 88906-072 Araranguá SC Brasil.

roger. ceccon@hotmail.com ${ }^{2}$ Programa de Pós-

Graduação em Saúde Coletiva, Universidade de Fortaleza. Fortaleza CE Brasil.

${ }^{3}$ Programa de Pós-

Graduação em Saúde

Coletiva, Universidade

Federal do Rio Grande do

Sul. Porto Alegre RS Brasil.

\begin{abstract}
This paper aims to identify sociodemographic and care characteristics of dependent older adults, formal and family caregivers in $\mathrm{mu}$ nicipalities from different Brazilian regions. A cross-sectional study was carried out with a sample of 175 people, of whom 64 were older adults, 27 formal caregivers, and 84 family caregivers. Semi-structured interviews were conducted with specific questions for each group on the theme of care and dependence. Most older adults were female, aged 80 years or older, with low education and have been dependent for four years or more. Older adults reported feelings of loneliness, pointed out difficulties in medical care, and 29\% had only access to Primary Health Care actions. Inequalities, burden, illnesses, and social problems were found among family caregivers. Black females with no formal employment, little or no training for the function, and low remuneration predominated among formal caregivers, and care was associated with domestic chores. We can conclude that gender and race inequalities persist in the care of dependent older adults, and we observed that the rigid social roles assigned to men and women in Brazil persist in the family and work dynamics in caring for the dependent older adults. Key words Aging, Frail older adult, Caregivers, Elderly health
\end{abstract}

Resumo $O$ artigo tem como objetivo identificar características sociodemográficas e assistenciais de idosos dependentes, cuidadores formais e familiares em municípios de diferentes regiões brasileiras. Realizou-se um estudo transversal com amostra de 175 pessoas, sendo 64 idosos, 27 cuidadores formais e 84 cuidadores familiares. Foram realizadas entrevistas semiestruturadas, com questões específicas para cada grupo sobre a temática do cuidado e dependência. A maioria dos idosos era do sexo feminino, com idade igual ou maior a 80 anos, com baixa escolaridade e dependente há quatro anos ou mais. Os idosos relataram sentimentos de solidão, apontaram dificuldades na assistência médica e 29\% tinham acesso apenas a ações da Atenção Primária à Saúde. Entre os cuidadores familiares, encontrou-se desigualdades, sobrecargas, adoecimentos e problemas sociais. Entre os formais predominou o sexo feminino, a raça negra, sem vínculo legal de trabalho, pouca ou nenhuma formação para a função e baixa remuneração; o cuidado associou-se aos afazeres domésticos. Conclui-se que perduram as desigualdades de gênero e raça no cuidado às pessoas idosas em situação de dependência. Observou-se também que a rigidez dos papeis sociais atribuidos a homens e mulheres no Brasil persistem nas dinâmicas familiares e laborais no cuidado ao idoso dependente.

Palavras-chave Envelhecimento, Idoso dependente, Cuidador, Saúde do idoso 


\section{Introdução}

Nas últimas décadas, o envelhecimento populacional foi marcado pelo aumento da expectativa de vida e redução das taxas de natalidade e mortalidade na maioria dos países do mundo. Houve crescimento da quantidade de idosos com 80 anos e mais, etapa vulnerável do ponto de vista social e da saúde física e mental, dentre as quais é comum a perda de autonomia e o aumento da dependência ${ }^{1}$.

A dependência é a incapacidade funcional dos idosos em realizar Atividades Básicas da Vida Diária (ABVD), como alimentar-se, vestir-se e tomar banho ${ }^{2}$ ou a impossibilidade de executar Atividades Instrumentais da Vida Diária (AIVD), como ir ao banco, pegar ônibus e comunicar-se ${ }^{3}$. Neste caso, os idosos necessitam de auxílio para a realização destas tarefas e para a gestão da própria vida ${ }^{4}$.

Na América Latina, 40\% dos idosos precisam de cuidados prolongados e este número triplicará nas próximas três décadas ${ }^{5}$. Em 2050, o Brasil terá cerca de 77 milhões de pessoas dependentes de cuidados, entre idosos e crianças ${ }^{6}$, e apenas $30 \%$ dos municípios possuíam instituições assistenciais de longa permanência no ano de 2009, a maioria na região sudeste do país ${ }^{7}$. Ao mesmo tempo em que aumenta a população longeva e dependente, persiste o déficit de cuidadores, profissionais e serviços de saúde preparados para assisti-los.

No Brasil, a maioria dos cuidadores são pessoas da família, mulheres (cônjuges ou filhas) com 50 anos ou mais e com proximidade física e afetiva com o idoso. O trabalho, muitas vezes, é ininterrupto e solitário, sem o apoio de serviços e políticas públicas de proteção para o desenvolvimento desta função. Os mesmos sofrem restrições em suas vidas pessoais, gerando sobrecarga, adoecimento, desemprego e afastamento da rede social e afetiva ${ }^{9,10}$.

No ano de 2002 a função de cuidador foi reconhecida como ocupação pelo Ministério do Trabalho e Renda no Brasil. Foi considerado "cuidador" o indivíduo que assiste e promove bem -estar, saúde, alimentação, higiene, educação, cultura e lazer à pessoa dependente. Entretanto, a profissão é marcada por precarização nos vínculos trabalhistas, falta de preparação específica, baixos salários e longas jornadas ${ }^{11}$. Apesar das políticas de proteção social ao idoso implementadas no país nas últimas décadas, a oferta de serviços pelo Estado restringe-se a ações específicas e pontuais de assistência à saúde, e atribui à fa- mília o compromisso pelo cuidado prolongado no âmbito do domicílio. Inexiste uma política específica que determine os papéis atribuídos à família e à rede de serviços públicos ${ }^{12}$, tornando vulnerável tanto o idoso quanto o cuidador.

$\mathrm{O}$ conceito de vulnerabilidade contribui para compreender a situação dos idosos e seus cuidadores, pois refere-se à garantia de cidadania de populações politicamente fragilizadas na perspectiva dos direitos humanos, resultado da combinação dos domínios individual, social e pragmático. A vulnerabilidade individual compreende os aspectos biológicos, emocionais, cognitivos e atitudinais; a social é caracterizada por aspectos culturais, sociais e econômicos que determinam as oportunidades de acesso a bens e serviços; e a vulnerabilidade programática refere-se aos recursos sociais necessários para a proteção do indivíduo a riscos à integridade e ao bem-estar físico, psicológico e social ${ }^{13}$.

Neste estudo, um conjunto de pessoas envolvidas no contexto da dependência e do cuidado ao idoso foi entrevistado, tendo em vista a necessidade de se conhecer in loco sua situação e vulnerabilidades. Assim, este estudo tem como objetivo identificar as características sociodemográficas e assistenciais de idosos dependentes, cuidadores familiares e cuidadores formais em oito municípios das diferentes regiões do Brasil.

\section{Método}

\section{Tipo de estudo}

Trata-se de um estudo transversal e descritivo realizado no ano de 2019 em oito municípios localizados nas cinco regiões brasileiras: Araranguá (SC), Brasília (DF), Fortaleza (CE), Manaus (AM), Porto Alegre (RS), Recife (PE), Rio de Janeiro (RJ) e Teresina (PI). Faz parte de uma pesquisa multicêntrica que estudou a situação de idosos com dependência física, mental/emocional, cognitiva ou social, cujo foco é subsidiar a elaboração de uma política pública que atenda ao idoso dependente e à pessoa que o cuida. Foi coordenada pela Fundação Oswaldo Cruz em parceria com instituições de ensino brasileiras ${ }^{14}$.

\section{Amostra}

Com amostra intencional, 175 pessoas participaram deste estudo, sendo 64 idosos com dependências, 27 cuidadores formais e 84 cuidadores familiares (Tabela 1). Considerou-se "idoso 
Tabela 1. Quantitativo de idosos dependentes, cuidadores familiares e cuidadores formais entrevistados na pesquisa, municípios de diferentes regiões do Brasil, 2019.

\begin{tabular}{lrrrrrr}
\hline \multirow{2}{*}{ Município/Estado } & \multicolumn{2}{c}{ Idosos dependentes } & \multicolumn{2}{c}{ Cuidadores familiares } & \multicolumn{2}{c}{ Cuidadores formais } \\
\cline { 2 - 6 } & $\mathbf{n}$ & $\mathbf{\%}$ & $\mathbf{n}$ & $\mathbf{\%}$ & $\mathbf{n}$ & $\%$ \\
\hline Araranguá (SC) & 12 & 18,8 & 13 & 15,5 & 6 & 22,2 \\
Belo Horizonte (BH) & 7 & 10,9 & 11 & 13,1 & 0 & 0,0 \\
Brasília (DF) & 10 & 15,6 & 10 & 11,9 & 0 & 0,0 \\
Fortaleza (CE) & 10 & 15,6 & 11 & 13,1 & 5 & 18,5 \\
Manaus (AM) & 5 & 7,8 & 10 & 11,9 & 5 & 18,5 \\
Porto Alegre (RS) & 11 & 17,2 & 11 & 13,1 & 4 & 14,8 \\
Rio de Janeiro (RJ) & 3 & 4,7 & 7 & 8,3 & 2 & 7,5 \\
Teresina (PI) & 6 & 9,4 & 11 & 13,1 & 5 & 18,5 \\
Total & 64 & 100 & 84 & 100 & 27 & 100 \\
\hline
\end{tabular}

dependente" a pessoa com 60 anos e mais que, pela redução ou falta de capacidade física ou cognitiva, tem necessidade de ajuda para a realização das ABVD ou AIVD, implicando na presença de, pelo menos, mais uma pessoa para exercer cuidado $^{15}$.

Considerou-se dependência física a incapacidade funcional, prática ou motora para realizar ABVD ou AIVD; e dependência cognitiva a perda completa ou parcial da orientação no tempo, da memória, atenção, realização de cálculo, linguagem e capacidade visual ${ }^{16}$. Os cuidadores foram compreendidos como formais (contratados) ou familiares, considerados como aqueles que prestam assistência ou cuidado ao idoso no exercício das atividades diárias ${ }^{17}$.

Foram excluídos os idosos que estavam em Instituições de Longa Permanência ou que residem sozinhos, por causa do objeto de estudo que previa compreender a situação da díade: idoso dependente-cuidador familiar.

\section{Coleta de dados}

Para a coleta de dados, os participantes foram identificados por meio das Secretarias Municipais de Saúde e dos serviços de Atenção Primária à Saúde (APS) de cada município envolvido no estudo. Em seguida, procedeu-se o contato e o agendamento das entrevistas com os interlocutores, todas realizadas em suas residências por entrevistadores previamente capacitados.

Os dados foram coletados por meio de entrevistas semiestruturadas, com itens que contemplaram a obtenção de dados quantitativos e qualitativos, utilizando-se um roteiro construído e consensualizado por um grupo de pesquisadores de diferentes universidades brasileiras. Foram utilizadas perguntas específicas para cada grupo entrevistado que envolveu a percepção acerca de temas relativos ao cuidado e à dependência.

As entrevistas foram gravadas em áudio (com permissão dos entrevistados) e transcritas. Destaca-se que as variáveis quantitativas selecionadas para este estudo foram coletadas do corpus textual gerado pelas transcrições realizadas pelos pesquisadores, contendo informações sociodemográficas e assistenciais relacionadas às práticas de cuidado e aspectos do mundo do trabalho.

\section{Variáveis}

As variáveis foram agrupadas de acordo com cada grupo de participante na pesquisa:

Idoso dependente:

Características sociodemográficas: sexo, raça, estado civil, faixa etária, quantidade de filhos e netos, religião, escolaridade, com quem reside, adaptação da residência às necessidades, cognição (capacidade preservada ou prejudicada de orientação no tempo, da memória, atenção, realização de cálculo, linguagem e capacidade visual) e convívio social (mantém convivência afetiva com familiares, amigos, vizinhos ou outros).

Características assistenciais: tempo que necessita de cuidado, cuidador profissional e médico que acompanha.

Cuidadores familiares:

Características sociodemográficas: sexo, raça/ cor e faixa etária;

Características assistenciais: grau de parentesco, motivo para ser cuidador, tempo na função, 
alternância nos cuidados, autopercepção sobre a própria saúde e problemas emocionais (Tristeza, estresse, cansaço, sobrecarga, insônia e irritação).

Cuidadores formais:

Características sociodemográficas: sexo, raça/ cor e faixa etária;

Características assistenciais e profissionais: contrato de trabalho, curso de formação, remuneração, tempo de atuação, realização de outras atividades no lar onde trabalha, dificuldades vivenciadas no trabalho, sentimento pela pessoa idosa e problemas emocionais (Tristeza, depressão, fadiga, sobrecarga e ansiedade) e sociais (Falta de reconhecimento, liberdade e relações sociais).

\section{Análise estatística}

As variáveis foram inseridas e categorizadas em um banco de dados no software Excel e codificadas. Posteriormente, para a análise estatística, utilizou-se o programa Statistical Package for the Social Sciences (SPSS), versão 20.0, e as variáveis foram apresentadas por meio de frequência bruta e relativa.

\section{Ética da pesquisa}

O projeto foi aprovado pelo Comitê de Ética e Pesquisa da Fundação Oswaldo Cruz. Todos os participantes assinaram o Termo de Consentimento Livre e Esclarecido.

\section{Resultados}

Foram entrevistados 64 idosos dependentes, 84 cuidadores familiares e 27 cuidadores formais em diferentes municípios brasileiros, constituindo uma amostra de 175 pessoas. Dentre os idosos, a maioria era mulher $(64,1 \%)$, da raça branca (56,3\%), com baixa escolaridade $(15,6 \%$ eram analfabetos e $40,6 \%$ possuíam ensino fundamental incompleto) e de religião católica (71,9\%). A maioria dos idosos possuía idade superior a 80 anos $(54,7 \%)$, viviam sem a presença de companheiro $(68,7 \%)$, tinham filhos $(87,6 \%)$ e netos (75\%). Do total, $37,5 \%$ moravam com a filha, $31,3 \%$ com companheiro e $70,3 \%$ em casa adaptada às necessidades (Tabela 2).

Dos idosos, 23,1\% necessitavam de cuidados há tempo maior ou igual a quatro anos; $87,7 \%$ não tinham cuidador profissional e 29,2\% eram acompanhados pelo médico da APS. A maioria referiu sentir mal-estar com a situação em que se
Tabela 2. Características sociodemográficas dos idosos dependentes de municípios em diferentes regiões do Brasil, 2019.

\begin{tabular}{|c|c|c|}
\hline Variável & $\begin{array}{c}\mathbf{n} \\
(64)\end{array}$ & $\%$ \\
\hline \multicolumn{3}{|l|}{ Sexo } \\
\hline Feminino & 41 & 64,1 \\
\hline Masculino & 23 & 35,9 \\
\hline \multicolumn{3}{|l|}{$\operatorname{Raça}^{*}$} \\
\hline Branca & 36 & 56,3 \\
\hline Negra (Parda e preta) & 20 & 31,2 \\
\hline \multicolumn{3}{|l|}{ Situação conjugal } \\
\hline Com companheiro & 24 & 37,5 \\
\hline Sem companheiro & 40 & 62,5 \\
\hline \multicolumn{3}{|l|}{ Faixa etária (anos) } \\
\hline $60-69$ & 6 & 9,4 \\
\hline $70-79$ & 23 & 35,9 \\
\hline$\geq 80$ & 35 & 54,7 \\
\hline \multicolumn{3}{|l|}{ Filhos $^{\star *}$} \\
\hline Nenhum & 2 & 3,1 \\
\hline $1-3$ & 28 & 43,8 \\
\hline $4-7$ & 17 & 26,6 \\
\hline$\geq 8$ & 11 & 17,2 \\
\hline \multicolumn{3}{|l|}{$\operatorname{Netos}^{\star * *}$} \\
\hline Nenhum & 5 & 7,8 \\
\hline $1-3$ & 23 & 36,0 \\
\hline $4-7$ & 10 & 15,6 \\
\hline$\geq 8$ & 15 & 23,4 \\
\hline \multicolumn{3}{|l|}{ Religião $^{* * * *}$} \\
\hline Católica & 46 & 71,9 \\
\hline Evangélica & 7 & 10,9 \\
\hline Outra & 6 & 9,4 \\
\hline \multicolumn{3}{|l|}{ Escolaridade ${ }^{* * * * *}$} \\
\hline Analfabeto & 10 & 15,6 \\
\hline Ensino Fundamental Incompleto & 26 & 40,6 \\
\hline Ensino Fundamental completo & 11 & 17,2 \\
\hline Ensino Médio & 8 & 12,5 \\
\hline Superior & 5 & 7,8 \\
\hline \multicolumn{3}{|l|}{ Com quem reside } \\
\hline Companheiro & 20 & 31,3 \\
\hline Filha & 24 & 37,5 \\
\hline Filho & 12 & 18,8 \\
\hline Sozinho & 4 & 6,2 \\
\hline Outros familiares & 4 & 6,2 \\
\hline \multicolumn{3}{|l|}{ Casa adaptada às necessidades $* * * * * *$} \\
\hline Não & 12 & 29,7 \\
\hline Sim & 45 & 70,3 \\
\hline \multicolumn{3}{|l|}{ Convívio social $^{* * * * * * *}$} \\
\hline $\begin{array}{l}\text { Não mantém relacionamentos } \\
\text { sociais }\end{array}$ & 34 & 53,2 \\
\hline Mantém relacionamentos sociais & 22 & 34,3 \\
\hline
\end{tabular}


encontrava, tinham a cognição preservada, porém não mantinham relações sociais (Tabela 3 ).

$\mathrm{Na}$ Tabela 4, é possível observar que a maioria dos cuidadores familiares era do sexo feminino $(84,5 \%)$, da raça/cor branca $(40,5 \%)$ e tinha entre 40 a 59 anos $(52,4 \%)$. Os que exerciam o cuidado dos idosos com maior frequência eram as "filhas", que relataram estarem nessa função justamente pelo fato de serem "filhas" ou por "não ter outra pessoa que cuide". Grande parte dos cuidadores assistiam aos idosos há mais de 2 anos e não dividiam o trabalho com outras pessoas. A maioria referiu que, no exercício dessa atividade têm passado por adoecimentos $(60,7 \%)$, problemas emocionais $(75,0 \%)$, problemas sociais $(75,0 \%)$ e relatou que a família constitui a principal rede de apoio que ajuda no cuidado com o idoso $(48,8 \%)$.

A Tabela 5 apresenta as características dos cuidadores formais. Foi possível observar que a maioria era mulher $(92,6 \%)$, de raça negra $(63,0 \%)$ e encontrava-se na faixa etária de 40 a 59 anos $(74,1 \%)$. Grande parte não tinha contrato de trabalho $(74,1 \%)$, nunca realizou curso de formação para a função $(77,8 \%)$, recebia aproximadamente um salário mínimo $(\leq \mathrm{R} \$ 1.000,00)$

Tabela 3. Características assistenciais dos idosos dependentes em municípios de diferentes regiões do Brasil, 2019.

\begin{tabular}{|c|c|c|}
\hline Variáveis & $\begin{array}{c}\mathrm{n} \\
(64)\end{array}$ & $\%$ \\
\hline \multicolumn{3}{|c|}{$\begin{array}{l}\text { Tempo que necessita de cuidado } \\
(\text { anos })^{\star}\end{array}$} \\
\hline$<1$ & 9 & 14,1 \\
\hline 1 a 3 & 9 & 14,1 \\
\hline$\geq 4$ & 15 & 23,4 \\
\hline \multicolumn{3}{|l|}{ Cuidador profissional } \\
\hline Não & 57 & 89,1 \\
\hline Sim & 7 & 10,9 \\
\hline \multicolumn{3}{|l|}{ Médico que acompanha ${ }^{* *}$} \\
\hline APS & 19 & 29,7 \\
\hline Especialista & 12 & 18,7 \\
\hline Nenhum & 11 & 17,2 \\
\hline Outro & 13 & 20,3 \\
\hline \multicolumn{3}{|l|}{ Percepção de si ${ }^{* * *}$} \\
\hline Bem estar & 18 & 28,1 \\
\hline Mal estar & 39 & 61,0 \\
\hline \multicolumn{3}{|l|}{ Cognição } \\
\hline Prejudicada & 23 & 35,9 \\
\hline Preservada & 41 & 64,1 \\
\hline
\end{tabular}

e atuava na função há menos de um ano (59,3\%). Nessa população, $74,1 \%$ realizaram outras ativi-

Tabela 4. Características sociodemográficas e assistenciais dos cuidadores familiares em municípios de diferentes regiões do Brasil, 2019.

\begin{tabular}{|c|c|c|}
\hline Variáveis & $\begin{array}{c}\mathbf{n} \\
(\mathbf{8 4})\end{array}$ & $\%$ \\
\hline \multicolumn{3}{|l|}{ Sexo } \\
\hline Feminino & 71 & 84,5 \\
\hline Masculino & 13 & 15,5 \\
\hline \multicolumn{3}{|l|}{$\operatorname{Raça}^{*}$} \\
\hline Branca & 34 & 40,5 \\
\hline Negra (Parda e preta) & 32 & 38,1 \\
\hline \multicolumn{3}{|l|}{ Faixa etária (anos) } \\
\hline $20-39$ & 7 & 8,3 \\
\hline $40-59$ & 44 & 52,4 \\
\hline $60-79$ & 12 & 14,3 \\
\hline$\geq 80$ & 21 & 25,0 \\
\hline \multicolumn{3}{|l|}{ Grau de parentesco } \\
\hline Companheiro(a) & 20 & 23,8 \\
\hline Filha & 39 & 46,4 \\
\hline Outros familiares & 25 & 29,8 \\
\hline \multicolumn{3}{|l|}{ Motivo por ser o cuidador } \\
\hline Não ter outra pessoa & 31 & 36,9 \\
\hline Por ser a esposa & 6 & 7,1 \\
\hline Por ser a filha & 23 & 27,4 \\
\hline Outro motivo & 24 & 28,6 \\
\hline \multicolumn{3}{|l|}{ Tempo na função (anos) $)^{* *}$} \\
\hline$\leq 1$ & 8 & 9,5 \\
\hline $2-5$ & 41 & 48,8 \\
\hline $6-9$ & 8 & 9,5 \\
\hline$\geq 10$ & 22 & 26,2 \\
\hline \multicolumn{3}{|c|}{ Cuidados alternados com outras } \\
\hline \multicolumn{3}{|l|}{ pessoas } \\
\hline Não & 46 & 54,8 \\
\hline Sim & 38 & 45,2 \\
\hline \multicolumn{3}{|c|}{ Adoecimento (autorreferido) } \\
\hline Não & 33 & 39,3 \\
\hline Sim & 51 & 60,7 \\
\hline \multicolumn{3}{|c|}{ Problemas emocionais (autorreferido) } \\
\hline Não & 21 & 25,0 \\
\hline Sim & 63 & 75,0 \\
\hline \multicolumn{3}{|l|}{ Problemas sociais } \\
\hline Não & 21 & 25,0 \\
\hline Sim & 63 & 75,0 \\
\hline \multicolumn{3}{|l|}{ Rede de apoio } \\
\hline APS & 13 & 15,5 \\
\hline Família & 41 & 48,8 \\
\hline Vizinhos/Amigos & 6 & 7,1 \\
\hline Não recebe apoio & 24 & 28,6 \\
\hline
\end{tabular}


Tabela 5. Características sociodemográficas, de trabalho, psicológicas e de cuidado dos cuidadores formais em municípios de diferentes regiões do Brasil, 2019.

\begin{tabular}{|c|c|c|}
\hline Variáveis & $\begin{array}{c}\mathbf{n} \\
(27)\end{array}$ & $\%$ \\
\hline \multicolumn{3}{|l|}{ Sexo } \\
\hline Feminino & 25 & 92,6 \\
\hline Masculino & 2 & 7,4 \\
\hline \multicolumn{3}{|l|}{ Raça } \\
\hline Branca & 10 & 37,0 \\
\hline Negra (Parda ou preta) & 17 & 63,0 \\
\hline \multicolumn{3}{|l|}{ Faixa etária (anos) } \\
\hline $30-39$ & 6 & 22,2 \\
\hline $40-59$ & 20 & 74,1 \\
\hline$>60$ & 1 & 3,7 \\
\hline \multicolumn{3}{|l|}{ Contrato de trabalho } \\
\hline Não & 20 & 74,1 \\
\hline $\operatorname{Sim}$ & 7 & 25,9 \\
\hline \multicolumn{3}{|l|}{ Curso para cuidador } \\
\hline Não & 21 & 77,8 \\
\hline $\operatorname{Sim}$ & 6 & 22,2 \\
\hline \multicolumn{3}{|l|}{ Remuneração $(\mathrm{R} \$)^{*}$} \\
\hline$\leq 1.000$ & 11 & 40,7 \\
\hline $1.100-2.000$ & 6 & 22,2 \\
\hline$\geq 2.100$ & 2 & 7,4 \\
\hline \multicolumn{3}{|l|}{ Tempo de atuação (anos) } \\
\hline$\leq 1$ & 16 & 59,3 \\
\hline $1-3$ & 5 & 18,5 \\
\hline$\geq 4$ & 6 & 22,2 \\
\hline \multicolumn{3}{|c|}{$\begin{array}{l}\text { Executa outras atividades na casa que } \\
\text { não o cuidado do idoso }{ }^{\star *}\end{array}$} \\
\hline Não & 4 & 14,8 \\
\hline $\operatorname{Sim}$ & 20 & 74,1 \\
\hline \multicolumn{3}{|c|}{ Enfrenta dificuldade no trabalho ${ }^{\star * *}$} \\
\hline Não & 10 & 37,0 \\
\hline $\operatorname{Sim}$ & 15 & 55,6 \\
\hline \multicolumn{3}{|l|}{ Percepção sobre o trabalho ${ }^{* * * *}$} \\
\hline Gosta do que faz & 17 & 63,0 \\
\hline $\begin{array}{l}\text { Sente-se sobrecarregado e } \\
\text { desvalorizado }\end{array}$ & 5 & 18,5 \\
\hline \multicolumn{3}{|l|}{ Problemas emocionais } \\
\hline Não & 15 & 55,6 \\
\hline Sim & 12 & 44,4 \\
\hline \multicolumn{3}{|l|}{ Rede de apoio ${ }^{\star * * * *}$} \\
\hline Família/Outros cuidadores & 17 & 63,0 \\
\hline Não recebe apoio & 7 & 25,9 \\
\hline
\end{tabular}

dades na residência do idoso e 55,6\% enfrentaram dificuldades no trabalho. A maioria referiu gostar do que faz, não sofria com problemas emocionais e contava com o apoio de familiares do idoso e de outros cuidadores.

\section{Discussão}

Este estudo identificou características sociodemográficas e assistenciais que indicam vulnerabilidades em idosos dependentes, cuidadores familiares e formais. Observou-se que os entrevistados são afetados por vulnerabilidades individuais, sociais e programáticas ${ }^{13}$, envolvendo aspectos que sugerem a precarização das condições de vida e saúde.

Com relação às vulnerabilidades individuais e sociais, evidencia-se que a maioria dos idosos é do sexo feminino, com idade maior ou igual a 80 anos e com baixa escolaridade. Essas mulheres necessitam de cuidado há muito tempo (quatro anos ou mais) e relataram sintomas depressivos, de tristeza e solidão. Embora tenham filhos e netos, reclamam que muitos não mantêm um convívio frequente com elas. Não possuem cuidador profissional e nem assistência regular à saúde.

Esses dados corroboram a projeção demográfica para o Brasil, que aponta maior proporção de mulheres entre as pessoas idosas, em decorrência da mortalidade diferencial por sexo, que afeta precocemente a população masculina ${ }^{18,19}$. Nas famílias, além de constituírem o grupo que mais sofre com a dependência ${ }^{20-22}$, elas também se caracterizam pelo cuidado que prestam a outros idosos ${ }^{23-25}$. É preciso lembrar que a população com 80 anos ou mais está aumentando no Brasil, e sendo a mais vulnerável aos vários tipos de dependência, é a que exige uma maior quantidade de cuidadores ${ }^{14}$.

Estudos apontam que a baixa escolaridade e as piores condições socioeconômicas dos idosos estão associadas à perda da capacidade física e funcional precocemente, pois essas pessoas tendem a acumular mais doenças ao longo da vida, desempenharam atividades laborais insalubres, possuem hábitos de vida prejudiciais e menor acesso aos serviços de saúde ${ }^{26-29}$. Além do mais, enfrentam maior dificuldade para receber ajuda, constituindo-se em um grupo extremamente vulnerável $^{29,30}$.

A solidão e o isolamento vivenciados pelos idosos são indicativos de perdas de convívio no âmbito familiar e social e, conforme Santini et al. ${ }^{31}$, podem ocasionar problemas emocionais e psicológicos, principalmente depressão. Essas questões que aparecem no estudo como queixas 
frequentes indicam a necessidade de atenção à saúde mental visando aumentar seus vínculos sociais, assim como tratar temáticas relacionadas à morte e à terminalidade ${ }^{32}$. A rede social de proteção, constituída por pessoas próximas, pode potencializar ou reduzir as vulnerabilidades?.

Embora os idosos entrevistados nesta pesquisa apresentem elevado número de filhos, netos e contem com a presença do cônjuge ou da pessoa que os cuida, muitos se sentem sozinhos. Este paradoxo pode ser resultado das mudanças e das dinâmicas familiares marcadas pela coexistência de bisnetos, netos e filhos na mesma residência, ao mesmo tempo em que se constata a perda de vínculo e de solidariedade ${ }^{33}$.

A vulnerabilidade programática encontrada neste estudo refere-se ao fato de que muitos idosos não contam com assistência médica e apenas $29 \%$ relataram acesso aos cuidados ofertados na APS. Esse achado reforça a hipótese acerca da necessidade de os serviços de saúde reconfigurarem suas práticas assistenciais para enfrentarem os desafios da multimorbidade e das necessidades associadas ao processo de envelhecimento. Necessitam-se estratégias que promovam acesso, reduzam a fragmentação do cuidado e valorizem e promovam as competências da APS para melhorar $o$ atendimento e, a partir dela, se dinamize uma nova organização das redes de atenção à saúde ${ }^{32,34,35}$. Não há solução: o número de idosos vai aumentar e é fundamental que o cuidado com essa população seja adequado e qualificado.

Com relação aos cuidadores familiares, foi possível identificar características que indicam vulnerabilidades individuais, emocionais e sociais. As condições em que vive este grupo denotam a precariedade da função de "cuidador", marcada por desigualdades, sobrecargas, adoecimentos e diversos problemas. A maioria são mulheres, principalmente filhas e esposas, corroborando outras pesquisas brasileiras, segundo as quais a pessoa que mais cuida geralmente é a filha, o que incide sobre sua vida econômicofinanceira, pois a empobrece (por não ser uma atividade remunerada), provoca-lhe sobrecarga por um trabalho ininterrupto, aumenta os riscos à saúde e seu isolamento social ${ }^{14,23,36}$. Muitas são também idosas, inclusive longevas, o que configura um contexto de pessoas idosas cuidando de idosos.

Embora uma parte das mulheres entrevistadas afirme que recebe apoio dos familiares no cuidado com seu familiar dependente, outra refere não possuir redes sociais. A rede e o apoio social são importantes como medidas de supor- te para o enfrentamento de problemas tanto do idoso como da pessoa que cuida ${ }^{10}$.

O cuidado familiar exercido majoritariamente por mulheres simboliza as desigualdades de gênero presentes na sociedade, historicamente constituídas por relações de poder assimétricas entre os sexos, cuja atividade de cuidar no âmbito privado tem sido função predominantemente feminina. Nesta pesquisa, parte das entrevistadas revelou que a condição de "ser filha" foi determinante para assumir o papel de cuidadora. Esse fato impacta na vida pessoal, profissional, social e afetiva da mesma, além de produzir efeitos na sociedade como um todo. Apesar das transformações dos arranjos familiares e o papel social das mulheres, o processo de envelhecimento populacional não está sendo acompanhado de mudanças na divisão sexual do trabalho de cuidar, a não ser em casos excepcionais, especialmente no âmbito familiar ${ }^{33}$.

Na maioria dos casos, observou-se ausência de alternância nos cuidados, como se a família entregasse de vez a assistência a uma única pessoa que arca, de um lado, com o conforto emocional de assistir seu ente querido, mas, de outro, com o ônus da sobrecarga de trabalho e da perda do convívio social e, por vezes, do emprego ${ }^{24,36}$. Vários estudiosos têm mostrado que essas pessoas são também mais suscetíveis aos agravos em sua saúde mental, na medida em que o tempo gasto com os cuidados do idoso são longos e ininterruptos $^{12}$, frequentemente elas dormem mal e se afastam do convívio social ${ }^{37}$.

Ressalta-se a importância da atenção à saúde do cuidador, no sentido de reduzir os fatores de risco que ele (mais corretamente ela) corre no exercício de sua atividade. Neste estudo, a presença de cuidador profissional foi pequena e inferior se comparada aos cuidadores familiares. Esse dado reforça o quanto o cuidado familiar e informal é predominante com o idoso dependente, corroborando com outros estudos brasileiros ${ }^{23,24,36}$ e precisam de apoio dos serviços públicos.

As características dos cuidadores formais também sugerem vulnerabilidades sociais, principalmente relacionadas ao mundo do trabalho. A maioria era do sexo feminino, de raça negra, sem vínculo trabalhista formal, com pouca ou nenhuma formação para o exercício profissional e recebe baixa remuneração. Além das condições precárias de trabalho, a maioria dos cuidadores formais acumulava outras atividades na residência onde cuida do idoso, como cozinhar, limpar a casa e cuidar dos demais membros da família. 
Dentre as vulnerabilidades dos cuidadores formais participantes deste estudo, estão a mão de obra predominantemente negra, com pouca qualificação e, na maioria das vezes, sem contrato de trabalho formal e garantias trabalhistas. A participação de mulheres negras e com baixa escolaridade no mercado de trabalho reflete a intersecção entre as desigualdades de gênero, raça e classe social presentes na sociedade. Elas constituem o grupo social mais desfavorecido e vulnerável, pois os regimes capitalistas, patriarcais e racistas impedem que as mesmas consigam melhores rendimentos e postos de trabalho, ficando sobrerrepresentadas nas ocupações de menor prestígio $^{38}$.

No Brasil, as taxas de escolarização dos negros são baixas e muitos ocupam trabalhos domésticos, precários e com menores remunerações quando comparados às pessoas brancas ${ }^{39}$. A ausência de contrato de trabalho formal e de formação para o exercício da profissão evidencia a falta de políticas públicas e sociais que garantam a qualificação dos cuidadores formais ${ }^{36}$. Isso ocorre, apesar de iniciativas governamentais, como o Programa Nacional de Acesso ao Ensino Técnico e Emprego (Pronatec), que oferece cursos técnicos para cuidadores de idosos. No entanto, se bem formadas, essas pessoas exigiriam melhores salários e vínculo trabalhista, o que, aliás, já é feito nos casos em que os cuidadores são organizados em cooperativas ou em pequenas empresas.

Este estudo, embora aborde aspectos fundamentais da díade idoso dependente-cuidador, apresenta algumas limitações. A coleta de dados é de natureza eminentemente qualitativa, a amostra é constituída por um pequeno número de pessoas e houve algumas não respostas às questões das entrevistas. Entretanto, os resultados identificam situações que são corroboradas por muitas outras pesquisas nacionais e internacionais aqui citadas.

\section{Conclusão}

As características sociodemográficas e assistenciais de uma amostra de idosos e cuidadores formais e familiares de diferentes municípios brasileiros identificadas nesta investigação constituem vulnerabilidades individuais, sociais e programáticas.

O estudo identificou fragilidades nos idosos decorrentes da situação de dependência. Além do mais, observou-se desigualdades de gênero e raça no cuidado às pessoas idosas, demonstrando a rigidez dos papeis sociais atribuídos aos homens e mulheres de acordo com a cor da pele na sociedade brasileira. No âmbito familiar, as redes de apoio demonstram relevância diante da temática estudada particularmente para prevenir agravos emocionais e sociais. E quanto aos cuidadores formais, há a necessidade de uma política de valorização e qualificação, contribuindo para a formalização da profissão.

Os dados apresentados são de uma amostra de idosos e cuidadores, não podendo ser generalizados e ou considerados representativos da sociedade brasileira. Entretanto, podem contribuir para a criação, o desenvolvimento e a implementação de estratégias governamentais e sociais para a melhoria e a ampliação das redes de apoio e a regulamentação de uma política e adequada que trate da dependência e do exercício do cuidar.

\section{Colaboradores}

RF Ceccon, LJES Vieira, CC Praça Brasil, KG Soares, VM Portes, CAS Garcia Júnior, IJC Schneider, AAF Carioca participaram igualmente da concepção, delineamento, análise e interpretação dos dados, b) redação do artigo ou a sua revisão crítica, e c) aprovação da versão a ser publicada. 


\section{Referências}

1. Freedman A, Nicolle J. Social isolation and loneliness: the new geriatric giants Approach for primary care. Can Fam Physician 2020; 66(3):176-182.

2. Del Duca GF, Silva MC, Halall PC. Incapacidade funcional para atividades básicas e instrumentais da vida diária em idosos. Rev Saude Publica 2009; 43(5):796805.

3. Alves LC, Leite IC, Machado CJ. Conceituando e mensurando a incapacidade funcional da população idosa: uma revisão de literatura. Cien Saude Colet 2008; 13(4):1199-207.

4. Klompstra L, Ekdahl AW, Krevers B, Milberg A, Eckerblad J. Factors related to health-related quality of life in older people with multimorbidity and high health care consumption over a two-year period. BMC Geriatr 2019; 19(1):187.

5. Organização Pan-americana de Saúde (OPAS). Plano de ação para a saúde da população idosa. Washington: OPAS; 2019.

6. Instituto Brasileiro de Geografia e Estatística (IBGE). Características gerais dos moradores 2012-2016. Rio de Janeiro: IBGE; 2017.

7. Camarano AA, organizador. Cuidados de Longa Duração para a População Idosa: um novo risco social a ser assumido? Rio de Janeiro: IPEA; 2010.

8. Greenwood N, Pound C, Brearley S, Smith R. A qualitative study of older informal carers' experiences and perceptions of their caring role. Maturitas 2019; (124):1-7.

9. Minayo MCS. Cuidar de quem cuida de idosos dependentes: Por uma política necessária e urgente. Cien Saude Colet 2020; 26(1):7-16.

10. Carmichael F, Ercolani M. Overlooked and undervalued: the caring contribution of older people. Int $J$ Soc Econ 2014; 41(5):397-419.

11. Lampert CDT, Scortegagna SA, Grzybovski D. Dispositivos legais no trabalho de cuidadores: aplicação em instituições de longa permanência. REAd 2016; 85(3):360-380.

12. Karsch UM. Idosos dependentes: famílias e cuidadores. Cad Saude Publica 2003; 19(3):861-866.

13. Ayres J, Calazans GJ, Saletti Filho HC, França Júnior I. Risco, vulnerabilidade e práticas de prevenção e promoção da saúde. In: Campos G, Minayo MCS, Akerman M, Drumond Júnior M, Carvalho YM, organizadores. Tratado de Saúde Coletiva. São Paulo: Editora Fiocruz; 2006. p. 375-417.

14. Minayo MCS. Estudo situacional dos idosos dependentes que residem com suas famílias visando subsidiar uma política de atenção e de apoio aos cuidadores. Rio de Janeiro: Fiocruz; 2019. (Projeto de Pesquisa).

15. Unión Europea (UE). Consejo de Europa. Recomendación 1591. Retos de la política social en las sociedades europeas que envejecen. Bruselas: Comisión Europea; 2003.

16. Brasil. Portaria no 2.528 , de 19 de outubro de 2006 . Aprova a Política Nacional de Saúde do Idoso. Diário Oficial da União 2006; 12 dez.

17. Bertolucci PH, Brucki SM, Campacci SR, Juliano Y. O Mini Exame do Estado Mental em uma população geral: Impacto da escolaridade. Arq Neuropsiquiatr 1994; 52(1):1-7.
18. Batista AS, Jaccoud LB, Aquino L, El-Moor PD. Envelhecimento e dependência: desafios para a organização da proteção social. Brasília: MPS, SPPS; 2008.

19. Instituto Brasileiro de Geografia e Estatística (IBGE). Tábua completa de mortalidade para o Brasil 2018: Breve análise da evolução da mortalidade no Brasil. Rio de Janeiro: IBGE; 2019.

20. Auais M, Ahmed T, Alvarado B, Phillips SP, Rosendaal N, Curcio CL, Fernandes J, Guralnik J, Zunzunegui MV. Gender differences in four-year incidence of self-reported and performance-based functional disability: The International Mobility in Aging Study. Arch Gerontol Geriatr 2019; 82:266-272.

21. Guerra RO, Alvarado BE, Zunzunegui MV. Life course, gender and ethnic inequalities in functional disability in a Brazilian urban elderly population. Aging Clin Exp Res 2008; 20(1):53-61.

22. Lima-Costa MF, Peixoto SV, Malta DC, Szwarcwald CL, Mambrini JVM. Informal and paid care for Brazilian older adults (National Health Survey, 2013). Rev Saude Publica 2017; 51(Supl. 1):6s.

23. Giacomin KC, Uchoa E, Lima-costa MFF. Projeto Bambuí: a experiência do cuidado domiciliário por esposas de idosos dependentes. Cad Saude Publica 2005; 21(5):1509-1518.

24. Diniz MAA, Melo BRS, Neri KH, Casemiro FG, Figueiredo LC, Gaioli CCLO, Gratão ACM. Estudo comparativo entre cuidadores formais e informais de idosos. Cien Saude Colet 2018; 23(11):3789-3798.

25. Duarte YAO, Berzins MAVS, Giacomin KC. Política Nacional do Idoso: as lacunas da lei e a questão dos cuidadores. In: Alcântara AO, Camarano AA, Giacomin KC, organizadores. Política Nacional do Idoso: velhas e novas questões. Rio de Janeiro: Ipea; 2016. p. 457-478.

26. Minayo MCS. O imperativo de cuidar da pessoa idosa dependente. Cien Saude Colet 2019; 24(1):247-252.

27. Melzer D, Izmirlian G, Leveille SG, Guralnik JM. Educational differences in the prevalence of mobility disability in old age: the dynamics of incidence, mortality, and recovery. J Gerontol B Psychol Sci Soc Sci 2001; 56(5):294-301.

28. Singh-Manoux A, Marmot M. Role of socialization in explaining social inequalities in health. Soc Sci Med 2005; 60(9):2129-2133.

29. Lima-Costa MF, Oliveira C, Macinko J, Marmot M. Socioeconomic inequalities in health in older adults in Brazil and England. Am J Public Health 2012; 102(8):1535-1541.

30. Lima-costa M, Mambrini JVM, Peixoto SV. Socioeconomic inequalities in activities of daily living limitations and in the provision of informal and formal care for noninstitutionalized older Brazilians: $\mathrm{Na}$ tional Health Survey, 2013. Int J Equity Health 2016; 15(1):137.

31. Santini ZI, Jose PE, Cornwell EY, Koyanagi A, Nielsen L, Hinrichsen C, Meilstrup C, Madsen KR, Koushede V. Social disconnectedness, perceived isolation, and symptoms of depression and anxiety among older Americans (NSHAP):a longitudinal mediation analysis. Lancet Public Health 2020; 5(1):e62-e70. 
32. Oliveira EB, Bozzetti MC, Hauser L, Duncan BB, Harzheim E. Avaliação da qualidade do cuidado a idosos nos serviços da rede pública de atenção primária à saúde de Porto Alegre, Brasil. Rev Bras Med Fam Comunidade 2013; 8(29):264-273.

33. Camarano AA. Quanto custa cuidar da população idosa dependente e quem paga por isto? In: Camarano AA, organizador. Novo regime demográfico: uma nova relação entre população e desenvolvimento? Rio de Janeiro: Ipea; 2014. p. 605-623.

34. Melo LA, Braga LC, Leite FPP, Bittar BF, Oséas JMF, Lima KC. Fatores associados à multimorbidade em idosos: uma revisão integrativa da literatura. Rev. Bras. Geriatr. Gerontol 2019; 22(1):1-11.

35. Nunes BP, Batista SRR, BOF DE Andrade F, Souzajunior PRB, Lima-Costa MF, Facchini LA. Multimorbidade em indivíduos com 50 anos ou mais de idade: ELSI-Brasil. Rev Saude Publica 2018; 52(2):1-12.

36. Giacomin KC, Duarte YAO, Camarano AA, Nunes DP, Fernandes D. Cuidado e limitações funcionais em atividades cotidianas - ELSI-Brasil. Rev Saude Publica 2018; 52(2):1-9.

37. Nascimento HG, Figueiredo AE. Demência, familiares cuidadores e serviços de saúde: o cuidado de si e do outro. Cien Saude Colet 2019; 24(4):1381-1392.

38. Lima M, Rios F, França D. Articulando gênero e raça: a participação das mulheres negras no mercado de trabalho (1995-2009). In: Marcondes MM, Pinheiro L, Queiroz C, Querino AC, Valverde D, organiadores. Dossiê mulheres negras: retrato das condições de vida das mulheres negras no Brasil. Brasília: Ipea; 2013. p. 53-80.

39. Instituto de Pesquisa Econômica Aplicada (IPEA). Situação social da população negra por estado. Brasília: Ipea; 2014.

Artigo apresentado em 12/05/2020

Aprovado em 11/08/2020

Versão final apresentada em 13/08/2020

Editores chefes: Maria Cecília de Souza Minayo, Romeu Gomes, Antônio Augusto Moura da Silva. Editora Associada da área de Saúde do Idoso: Joselia Oliveira Araújo Firmo 